Result(s)* Time taken for performing the surgery was 102 minutes. Frozen section of the left adnexal mass was reported as serous Borderline Ovarian tumour. Cytology of the peritoneal washing collected was reported to be negative for malignant cells. Pathological examination of the specimen showed Atypical proliferative serous tumour/serous borderline ovarian tumour of left ovary with surface involvement- FIGO stage I C2. Uterus had multiple leiomyoma. The pelvic nodes, omental tissue and the peritoneal tisssues were negative for implants or malignancy. The post- operative period was uneventful, and the patient was discharged on postoperative day 2 .

Conclusion* Robot Assisted Laparoscopic staging surgery can be performed safely in selected cases by well-trained surgeons without compromising the oncological outcomes.

\section{RESOLUTION AND PERSISTENCE OF OVARIAN CYSTS USING CYST DIAMETER IN ORDER TO RELATE FINDINGS TO THE PRACTICING PHYSICIAN}

${ }^{1} \mathrm{~A}$ Lasher*, ${ }^{2} \mathrm{~L}$ Harris, ${ }^{3} \mathrm{~J}$ Vannagell, ${ }^{3} \mathrm{E}$ Pavlik. ' University of Kentucky, College of Medicine, Lexington, USA; ${ }^{2}$ University Of Kentucky, College of Medicine, Lexington, USA; ${ }^{3}$ University of Kentucky, Gynecologic Oncology, Lexington, USA

\subsection{6/ijgc-2021-ESGO.504}

Introduction/Background* Ovarian cysts frequently resolve on their own, but little information on resolution is available for use in clinical practice. The objective of this study was to characterize the resolution of incident ovarian cysts in relation to cyst diameter, structure, age, body habitus, and menopausal status. These categorizations are important for decisions on whether to continue monitoring the cyst or intervene surgically.

Methodology A total of 2,638 women with incident cysts were selected who had undergone 51,356 transvaginal ultrasound examinations in the University of Kentucky Ovarian Cancer Screening Program and were negative for ovarian malignancy. Prevalent cysts were excluded as they would be examined with an undefined course of first appearance when defining resolution time. Other exclusions included women with concurrent ovarian malignancies, cysts with solid components, and cases where surgery interrupted the natural history of measured cysts. The descriptor reported here is cyst diameter used to relate findings to physicians discovering these structures by bimanual examination. The variables collected in this study were: age, BMI, cyst diameter, cyst structure (loculation/septation), family history of ovarian cancer, and use of hormone replacement therapy. All images were reviewed by a physician. Methods of analysis include t-tests, chi-square, and Cox regression analysis of cyst resolution times.

Result(s)* Women with a history of 1 or more cysts were included so that 2,465 unilocular and 1,420 septated cysts were available for analysis. Septated cysts (mean \pm SEM, 3.8 $\pm 0.03)$ had a larger diameter than unilocular cysts $(3.4$ $\pm 0.03), P<0.05$. The resolution of incident septated cysts was faster than unilocular cysts. Unilocular cysts, but not septated cysts, resolved the quickest in women $70+$ years of age, and resolution was the most prolonged in women $<40$ years. No difference in resolution was observed between pre- and postmenopausal status nor BMI in the resolution of unilocular or septated cysts.

Conclusion* While septated cysts tended to have a larger diameter than unilocular cysts, they were observed to resolve more rapidly. Menopausal status and body mass index were

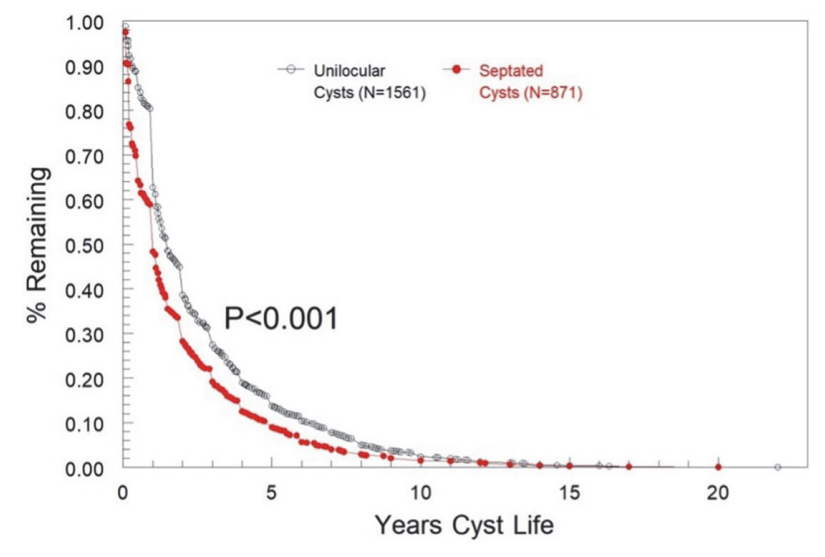

Abstract 1013 Figure 1

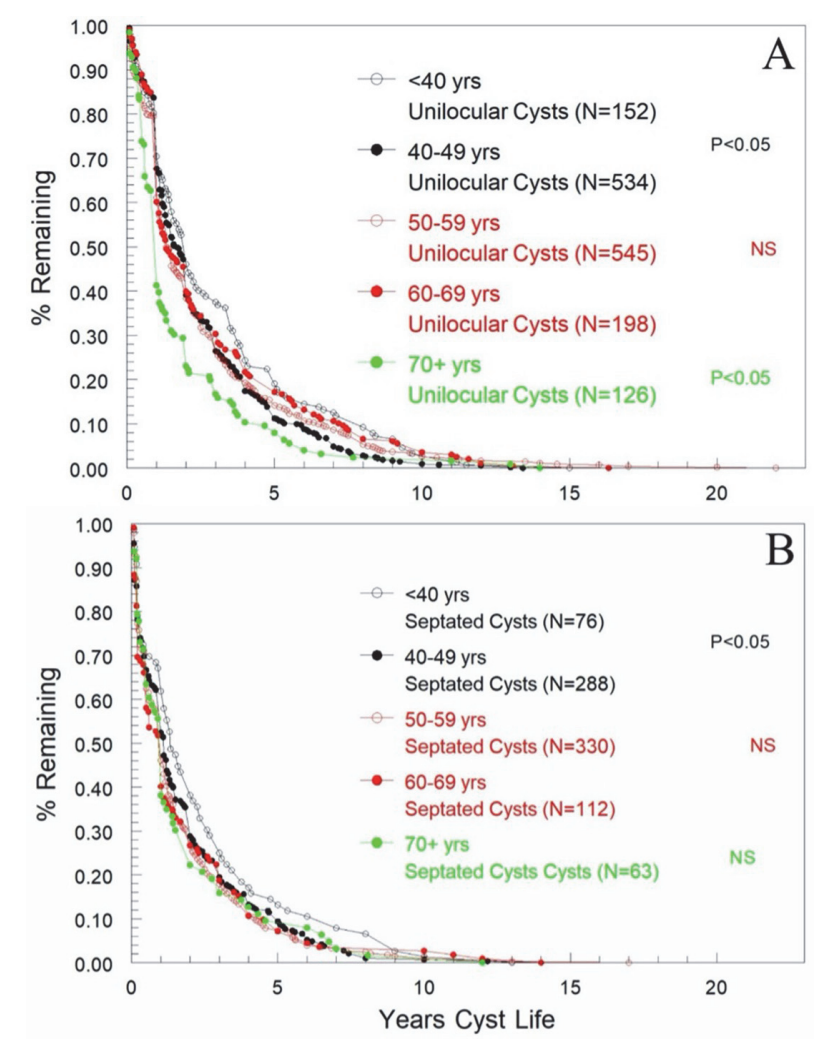

Abstract 1013 Figure 2

not seen to have an influence on the time to resolution of unilocular and septated cysts. For the clinician, this study establishes expectations for cyst resolution based on a primary estimation of cyst diameter in a population where no malignancy was observed.

\section{LINEAR RELATIONSHIP OF PERITONEAL CANCER INDEX AND SURVIVAL IN PATIENTS WITH EPITHELIAL OVARIAN CANCER IN CARCINOMATOSIS}

A Roosen, C Sanson*, M Faron, A Maulard, P Pautier, A Leary, C Chargari, C Genestie, F Zaccarini, S Scherier, P Morice, S Gouy. Gustave Roussy, Surgery, Villejuif, France

10.1136/ijgc-2021-ESG0.505 
Introduction/Background* The aim of this study was to investigate the relation between the PCI and overall survival (OS) and recurrence-free survival (RFS). The peritoneal cancer index (PCI) is one of the main prognostic factor for the evaluation of ovarian peritoneal carcinosis. Different thresholds have been reported in terms of prognosis and to help in the decision between chemotherapy or primary surgery, but no consensus was found.

Methodology Patients treated at Gustave Roussy between 2004 and 2017 for advanced epitoneal ovarian cancer in complete resection were included. The correlation between PCI and survival was studied using statistical modeling. Multivariate analysis was performed by a logistic regression model.

Result(s)* Of the 351 patients included, 27\% had initial surgery, $73 \%$ had interval surgery. The median follow-up was 52.7 months. The mean PCI was 10.8 (0-32). The linear model best represented the relationship between PCI and OS. Patients with neoadjuvant chemotherapy had a greater instantaneous risk of baseline death than those with initial surgery, as well as a more rapid increase in this risk as PCI increased. OS and PFS were better in the initial surgery group (103.4 months [79.1-NA] vs. 66.5 months [59.1-95.3] and 31.8 months [23.7-48.7] vs. 25.9 months [23.2-29] respectively). Risk factors for death were BMI, PCI and performance of neoadjuvant chemotherapy.

Conclusion* PCI is a major prognostic element but its linear relationship with survival does not allow us to establish a cutoff. Moreover, the prognostic impact of PCI is even stronger in the case of primary chemotherapy.

\section{REVISITING THE ROTTERDAM REGIME IN 2021: THE IMPACT OF CISPLATIN AND ORAL ETOPOSIDE IN REFRACTORY OVARIAN CANCER IN THE ERA OF GREAT ANTIEMETICS}

${ }^{1} \mathrm{M}$ Godfrey*, ${ }^{1} \mathrm{E}$ Khoury, ${ }^{1} \mathrm{~N}$ Martynyuk, ${ }^{1} \mathrm{~F}$ Gardner, ${ }^{2} \mathrm{C}$ Yeoh. ${ }^{1}$ Portsmouth University Hospital, Gynae Oncology, UK; ${ }^{2}$ University of Portsmouth, Molecular Biology, Portsmouth, UK

\subsection{6/ijgc-2021-ESG0.506}

Introduction/Background* Weekly Cisplatin plus oral daily Etoposide (the Rotterdam regime (RR), 2002) has been shown to be effective in platinum refractory (PR) ovarian cancer (OC), with a documented response rate of $46 \%$, with $29 \%$ complete response and overall survival (OS) of 13 months ${ }^{1}$. The RR is schedule intensive and can be difficult to tolerate, therefore may be underutilised as an option for patients with PR OC who have maintained a good performance status (PS). We are reporting our series of patients undergoing the RR with regards to efficacy and tolerability.

Methodology Retrospective case series $(n=13)$ with PR OC, and still fit $(\mathrm{PS}<2)$, who have previously been treated with multiple chemotherapy lines were assessed for tolerability, response and subsequent reversal back to platinum-sensitivity (P-S).

Result(s)* Ten had serous, 2 had clear cell carcinoma and 1 had carcinosarcoma of OC. The patients were heavily pretreated, with $15 \% 3^{\text {rd }}$ line, $55 \% 4^{\text {th }}$ line, $25 \% 5^{\text {th }}$ line, and $5 \% 7^{\text {th }}$ line cancer treatment. Five women have died of OC, all within one year of starting the RR. Three patients have been converted back to P-S disease post-RR (23\%), with one patient now alive 5 years after completing the RR. Of the remaining 5 women: Re-challenge with platinum $(n=2)$; referred for a clinical trial $(\mathrm{n}=1)$, awaiting surgery for single site disease $(n=1)$; still on the RR $(n=1)$.

Nausea and tiredness were the main side effects affecting $80 \%$ of all patients in their first 6 weeks. The RR had an improvised protocol with 4 antiemetics, and Magnesium sachet supplement. More than $70 \%$ had low Magnesium; and required supplement up to 5 weeks from completion of the last Infusion of Cisplatin. Tiredness was common and linked to low moods, which led to us setting up a 'buddy-system' for those about to embark on RR.

Conclusion* The RR converted $23 \%$ of PR OC back to P-S, with further results awaited. One patient is now in her sixthyear post-RR and has continued to have P-S disease. Despite significant side effects nearly half of patients maintained a $\mathrm{PS}<2$, therefore we advise consideration of using the $\mathrm{RR}$ in PR disease.

\section{THE APPLICATION OF J-PLASMA IN THE EXCISION OF DIAPHRAGMATIC LESIONS AS PART OF COMPLETE CYTOREDUCTION IN PATIENTS WITH ADVANCED OVARIAN CANCER}

V Theodoulidis*, A Prodromidou, V Pergialiotis, DE Vlachos, C Theofanakis, N Thomakos, A Rodolakis, D Haidopoulos. ALEXANDRA HOSPITAL ATHENS GREECE, First Department of Obstetrics and Gynecology National University of Athens

\subsection{6/ijgc-2021-ESG0.507}

Introduction/Background* The objective of this study was to investigate the safety and treatment efficacy of J-Plasma in cases of advanced Epithelial Ovarian Cancer with upper abdominal involvement undergoing peritoneal and diaphragmatic stripping.

Methodology A retrospective review of a prospectively maintained database of patients who had diaphragmatic stripping with the use of J-Plasma ${ }^{\circledR}$ from January 2016 to September 2019, due to peritoneal dissemination for advanced stage ovarian cancer (FIGO stage $\geq$ III) was performed.

Result(s)* A total of 12 patients who underwent diaphragmatic stripping with the use of J-plasma for cytoreduction due to advanced ovarian cancer were included. Baseline patients characteristics are shown at table 1 . The type of surgical procedure, median operative time, median estimated blood loss, time for the diaphragmatic resection, median length of hospital stay and postoperative complications are shown at table 2 . No major intraoperative complications were recorded including J-plasma related. No defect in the diaphragmatic integrity and connection with the pleural cavity following resection were detected as evaluated by the "bubble test". During a median follow-up period of 12 months (range:6-26 months), 2 patients (17\%) presented with a disease recurrence (one local pelvic wall recurrence and one distant peritoneal) while none of the patients died of the disease during the follow-up period. Median disease free survival was 12 months (range:1013 months)

Conclusion* J-plasma can be used during diaphragmatic stripping as it is associated with low rates of short-term morbidity and less operative time needed for stripping which are lower compared to the one that follows traditional peritoneal technic. Furthermore, in terms of disease control it seems to be particularly effective as none of patients experienced disease relapse in abdominal regions that were treated with $\mathrm{J}$ plasma. 